\title{
Clustering Via Decision Tree Construction
}

\author{
Bing Liu ${ }^{1}$, Yiyuan Xia ${ }^{2}$, and Philip S. Yu ${ }^{3}$ \\ 1 Department of Computer Science, University of Illinois at Chicago, $851 \mathrm{~S}$. \\ Morgan Street, Chicago, IL 60607-7053. liub@cs.uic.edu \\ 2 School of Computing, National University of Singapore, 3 Science Drive 2, \\ Singapore 117543. \\ 3 IBM T. J. Watson Research Center, Yorktown Heights, NY 10598. \\ psyu@us.ibm.com
}

Clustering is an exploratory data analysis task. It aims to find the intrinsic structure of data by organizing data objects into similarity groups or clusters. It is often called unsupervised learning because no class labels denoting an a priori partition of the objects are given. This is in contrast with supervised learning (e.g., classification) for which the data objects are already labeled with known classes. Past research in clustering has produced many algorithms. However, these algorithms have some shortcomings. In this paper, we propose a novel clustering technique, which is based on a supervised learning technique called decision tree construction. The new technique is able to overcome many of these shortcomings. The key idea is to use a decision tree to partition the data space into cluster (or dense) regions and empty (or sparse) regions (which produce outliers and anomalies). We achieve this by introducing virtual data points into the space and then applying a modified decision tree algorithm for the purpose. The technique is able to find clusters in large high dimensional spaces efficiently. It is suitable for clustering in the full dimensional space as well as in subspaces. It also provides easily comprehensible descriptions of the resulting clusters. Experiments on both synthetic data and real-life data show that the technique is effective and also scales well for large high dimensional datasets.

\section{Introduction}

Clustering aims to find the intrinsic structure of data by organizing objects (data records) into similarity groups or clusters. Clustering is often called unsupervised learning because no classes denoting an a priori partition of the objects are known. This is in contrast with supervised learning, for which the data records are already labeled with known classes. The objective of supervised learning is to find a set of characteristic descriptions of these classes. 
In this paper, we study clustering in a numerical space, where each dimension (or attribute) has a bounded and totally ordered domain. Each data record is basically a point in the space. Clusters in such a space are commonly defined as connected regions in the space containing a relatively high density of points, separated from other such regions by a region containing a relatively low density of points [12].

Clustering has been studied extensively in statistics [5], pattern recognition [16], machine learning [15], and database and data mining (e.g., $[25,32,7,14,1,2,3,8,10,11,20,21,22,23,29,30,31])$. Existing algorithms in the literature can be broadly classified into two categories [24]: partitional clustering and hierarchical clustering. Partitional clustering determines a partitioning of data records into $k$ groups or clusters such that the data records in a cluster are more similar or nearer to one another than the data records in different clusters. Hierarchical clustering is a nested sequence of partitions. It keeps merging the closest (or splitting the farthest) groups of data records to form clusters.

In this paper, we propose a novel clustering technique, which is based on a supervised learning method called decision tree construction [26]. The new technique, called CLTree (CLustering based on decision Trees), is quite different from existing methods, and it has many distinctive advantages. To distinguish from decision trees for classification, we call the trees produced by CLTree the cluster trees.

Decision tree building is a popular technique for classifying data of various classes (at least two classes). Its algorithm uses a purity function to partition the data space into different class regions. The technique is not directly applicable to clustering because datasets for clustering have no pre-assigned class labels. We present a method to solve this problem.

The basic idea is that we regard each data record (or point) in the dataset to have a class $Y$. We then assume that the data space is uniformly distributed with another type of points, called non-existing points. We give them the class, $N$. With the $N$ points added to the original data space, our problem of partitioning the data space into data (dense) regions and empty (sparse) regions becomes a classification problem. A decision tree algorithm can be applied to solve the problem. However, for the technique to work many important issues have to be addressed (see Section 2). The key issue is that the purity function used in decision tree building is not sufficient for clustering.

We use an example to show the intuition behind the proposed technique. Figure 1(A) gives a 2-dimensional space, which has 24 data $(Y)$ points represented by filled rectangles. Two clusters exist in the space. We then add some uniformly distributed $N$ points (represented by "o") to the data space (Figure 1(B)). With the augmented dataset, we can run a decision tree algorithm to obtain a partitioning of the space (Figure 1(B)). The two clusters are identified.

The reason that this technique works is that if there are clusters in the data, the data points cannot be uniformly distributed in the entire space. 


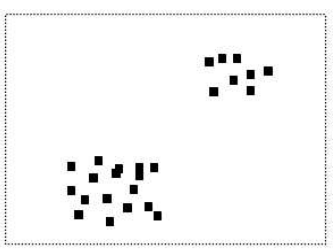

(A)

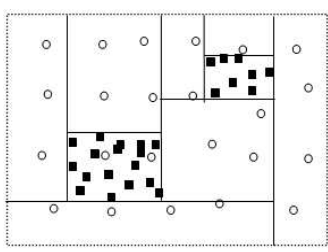

(B)

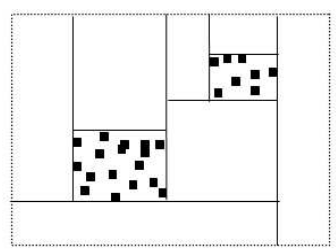

(C)

Fig. 1. Clustering using decision trees: an intuitive example

By adding some uniformly distributed $N$ points, we can isolate the clusters because within each cluster region there are more $Y$ points than $N$ points. The decision tree technique is well known for this task.

We now answer two immediate questions: (1) how many $N$ points should we add, and (2) can the same task be performed without physically adding the $N$ points to the data? The answer to the first question is that it depends. The number changes as the tree grows. It is insufficient to add a fixed number of $N$ points to the original dataset at the beginning (see Section 2.2). The answer to the second question is yes. Physically adding $N$ points increases the size of the dataset and also the running time. A subtle but important issue is that it is unlikely that we can have points truly uniformly distributed in a very high dimensional space because we would need an exponential number of points [23]. We propose a technique to solve the problem, which guarantees the uniform distribution of the $N$ points. This is done by not adding any $N$ point to the space but computing them when needed. Hence, CLTree can produce the partition in Figure $1(\mathrm{C})$ with no $N$ point added to the original data.

The proposed CLTree technique consists of two steps:

1. Cluster tree construction: This step uses a modified decision tree algorithm with a new purity function to construct a cluster tree to capture the natural distribution of the data without making any prior assumptions.

2. Cluster tree pruning: After the tree is built, an interactive pruning step is performed to simplify the tree to find meaningful/useful clusters. The final clusters are expressed as a list of hyper-rectangular regions.

The rest of the paper develops the idea further. Experiment results on both synthetic data and real-life application data show that the proposed technique is very effective and scales well for large high dimensional datasets.

\subsection{Our contributions}

The main contribution of this paper is that it proposes a novel clustering technique, which is based on a supervised learning method [26]. It is fundamentally different from existing clustering techniques. Existing techniques 
form clusters explicitly by grouping data points using some distance or density measures. The proposed technique, however, finds clusters implicitly by separating data and empty (sparse) regions using a purity function based on the information theory (the detailed comparison with related work appears in Section 5). The new method has many distinctive advantages over the existing methods (although some existing methods also have some of the advantages, there is no system that has all the advantages):

- CLTree is able to find clusters without making any prior assumptions or using any input parameters. Most existing methods require the user to specify the number of clusters to be found and/or density thresholds (e.g., $[25,32,21,7,14,1,2,3,10,23])$. Such values are normally difficult to provide, and can be quite arbitrary. As a result, the clusters found may not reflect the "true" grouping structure of the data.

- CLTree is able to find clusters in the full dimension space as well as in any subspaces. It is noted in [3] that many algorithms that work in the full space do not work well in subspaces of a high dimensional space. The opposite is also true, i.e., existing subspace clustering algorithms only find clusters in low dimension subspaces $[1,2,3]$. Our technique is suitable for both types of clustering because it aims to find simple descriptions of the data (using as fewer dimensions as possible), which may use all the dimensions or any subset.

- It provides descriptions of the resulting clusters in terms of hyper-rectangle regions. Most existing clustering methods only group data points together and give a centroid for each cluster with no detailed description. Since data mining applications typically require descriptions that can be easily assimilated by the user as insight and explanations, interpretability of clustering results is of critical importance.

- It comes with an important by-product, the empty (sparse) regions. Although clusters are important, empty regions can also be useful. For example, in a marketing application, clusters may represent different segments of existing customers of a company, while the empty regions are the profiles of non-customers. Knowing the profiles of non-customers allows the company to probe into the possibilities of modifying the services or products and/or of doing targeted marketing in order to attract these potential customers. Sparse regions also reveal outliers and anomalies, which are important for many applications.

- It deals with outliers effectively. Outliers are data points in a relatively empty region. CLTree is able to separate outliers from real clusters because it naturally identifies sparse and dense regions. When outliers are concentrated in certain areas, it is possible that they will be identified as small clusters. If such outlier clusters are undesirable, we can use a simple threshold on the size of clusters to remove them. However, sometimes such small clusters can be very useful as they may represent exceptions (or un- 
expected cases) in the data. The interpretation of these small clusters is dependent on applications.

\section{Building Cluster Trees}

This section presents our cluster tree algorithm. Since a cluster tree is basically a decision tree for clustering, we first review the decision tree algorithm in [26]. We then modify the algorithm and its purity function for clustering.

\subsection{Decision tree construction}

Decision tree construction is a well-known technique for classification [26]. A database for decision tree classification consists of a set of data records, which are pre-classified into $q(\geq 2)$ known classes. The objective of decision tree construction is to partition the data to separate the $q$ classes. A decision tree has two types of nodes, decision nodes and leaf nodes. A decision node specifies some test on a single attribute. A leaf node indicates the class.

From a geometric point of view, a decision tree represents a partitioning of the data space. A serial of tests (or cuts) from the root node to a leaf node represents a hyper-rectangle. For example, the four hyper-rectangular regions in Figure 2(A) are produced by the tree in Figure 2(B). A region represented by a leaf can also be expressed as a rule, e.g., the upper right region in Figure 2 (A) can be represented by $X>3.5, Y>3.5 \rightarrow O$, which is also the right most leaf in Figure 2(B). Note that for a numeric attribute, the decision tree algorithm in [26] performs binary split, i.e., each cut splits the current space into two parts (see Figure 2(B)).

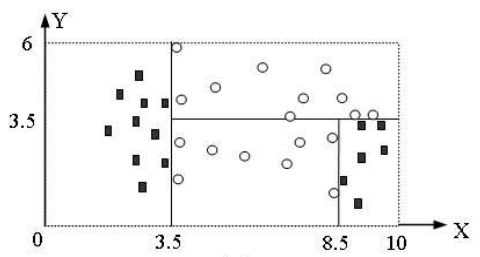

(A)

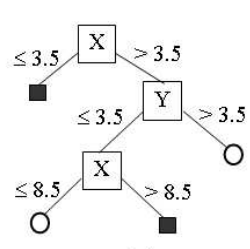

(B)

Fig. 2. An example partition of the data space and its corresponding decision tree

The algorithm for building a decision tree typically uses the divide and conquer strategy to recursively partition the data to produce the tree. Each successive step greedily chooses the best cut to partition the space into two 
parts in order to obtain purer regions. A commonly used criterion (or purity function) for choosing the best cut is the information gain $[26]^{4}$.

The information gain criterion is derived from information theory. The essential idea of information theory is that the information conveyed by a message depends on its probability and can be measured in bits as minus the logarithm to base 2 of that probability.

Suppose we have a dataset $D$ with $q$ classes, $C_{1}, \ldots, C_{q}$. Suppose further that we have a possible test $x$ with $m$ outcomes that partitions $D$ into $m$ subsets $D_{1}, \ldots, D_{m}$. For a numeric attribute, $m=2$, since we only perform binary split. The probability that we select one record from the set $D$ of data records and announce that it belongs to some class $C_{j}$ is given by:

$$
\frac{\operatorname{freq}\left(C_{j}, D\right)}{|D|}
$$

where $\operatorname{freq}\left(C_{j}, D\right)$ represents the number of data records (points) of the class $C_{j}$ in $D$, while $|D|$ is the total number of data records in $D$. So the information that it conveys is:

$$
-\log _{2}\left(\frac{\operatorname{freq}\left(C_{j}, D\right)}{|D|}\right) \text { bits }
$$

To find the expected information needed to identify the class of a data record in $D$ before partitioning occurs, we sum over the classes in proportion to their frequencies in $D$, giving:

$$
\operatorname{info}(D)=-\sum_{j=1}^{q} \frac{\operatorname{freq}\left(C_{j}, D\right)}{|D|} \times \log _{2}\left(\frac{\operatorname{freq}\left(C_{j}, D\right)}{|D|}\right)
$$

Now, suppose that the dataset $D$ has been partitioned in accordance with the $m$ outcomes of the test $X$. The expected amount of information needed to identify the class of a data record in $D$ after the partitioning had occurred can be found as the weighted sum over the subsets, as:

$$
\operatorname{info}_{X}(D)=-\sum_{i=1}^{m} \frac{\left|D_{i}\right|}{|D|} \times \operatorname{info}\left(D_{i}\right)
$$

where $\left|D_{i}\right|$ represents the number of data records in the subset $D_{i}$ after the partitioning had occurred. The information gained due to the partition is:

$$
\operatorname{gain}(X)=\operatorname{info}(D)-\operatorname{info}_{X}(D)
$$

Clearly, we should maximize the gain. The gain criterion is to select the test or cut that maximizes the gain to partition the current data (or space).

The procedure for information gain evaluation is given in Figure 3. It evaluates every possible value (or cut point) on all dimensions to find the cut point that gives the best gain.

\footnotetext{
${ }^{4}$ In [26], it can also use the information gain ratio criterion, which is the normalized gain. The normalization is used to avoid favoring a categorical attribute that has many values. Since we have no categorical attribute, this is not a problem.
} 
1 for each attribute $A_{i} \in\left\{A_{1}, A_{2}, A_{d}\right\}$ do

/* $^{*} A_{1}, A_{2}$, and $A_{d}$ are the attributes of $D * /$

2 for each value $x$ of $A_{i}$ in $D$ do

/* each value is considered as a possible cut */

3 compute the information gain at $x$

4 end

5 end

6 Select the test or cut that gives the best information gain to partition the space

Fig. 3. The information gain evaluation

Scale-up decision tree algorithms: Traditionally, a decision tree algorithm requires the whole data to reside in memory. When the dataset is too large, techniques from the database community can be used to scale up the algorithm so that the entire dataset is not required in memory. [4] introduces an interval classifier that uses data indices to efficiently retrieve portions of data. SPRINT [27] and RainForest [18] propose two scalable techniques for decision tree building. For example, RainForest only keeps an AVC-set (attribute-value, classLabel and count) for each attribute in memory. This is sufficient for tree building and gain evaluation. It eliminates the need to have the entire dataset in memory. BOAT [19] uses statistical techniques to construct the tree based on a small subset of the data, and correct inconsistency due to sampling via a scan over the database.

\subsection{Building cluster trees: Introducing $N$ points}

We now present the modifications made to the decision tree algorithm in [26] for our clustering purpose. This sub-section focuses on introducing $N$ points. The next sub-section discusses two changes that need to be made to the decision tree algorithm. The final sub-section describes the new cut selection criterion or purity function.

As mentioned before, we give each data point in the original dataset the class $Y$, and introduce some uniformly distributed "non-existing" $N$ points. We do not physically add these $N$ points to the original data, but only assume their existence.

We now determine how many $N$ points to add. We add a different number of $N$ points at each node in tree building. The number of $N$ points for the current node $E$ is determined by the following rule (note that at the root node, the number of inherited $N$ points is 0 ):

1 If the number of $N$ points inherited from the parent node of $E$ is less than the number of $Y$ points in $E$ then

2 the number of $N$ points for $E$ is increased to the number of $Y$ points in $E$

3 else the number of inherited $N$ points from its parent is used for $E$ 
Figure 4 gives an example. The (parent) node $P$ has two children nodes $L$ and $R$. Assume $P$ has $1000 Y$ points and thus $1000 N$ points, stored in $P . Y$ and P.N respectively. Assume after splitting, $L$ has $20 Y$ points and $500 \mathrm{~N}$ points, and $R$ has $980 Y$ points and $500 N$ points. According to the above rule, for subsequent partitioning, we increase the number of $N$ points at $R$ to 980. The number of $N$ points at $L$ is unchanged.

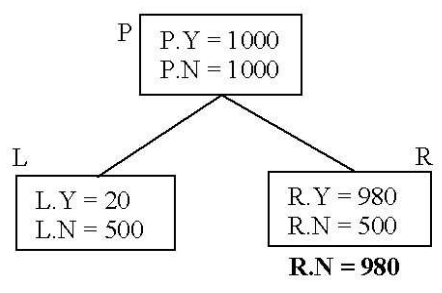

Fig. 4. Distributing $N$ points

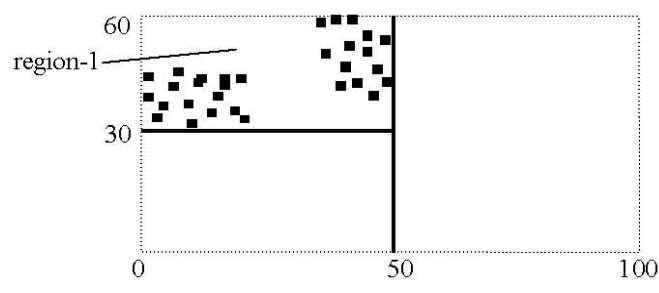

Fig. 5. The effect of using a fixed number of $N$ points

The basic idea is that we use an equal number of $N$ points to the number of $Y$ (data) points (in fact, 1:1 ratio is not necessary, see Section 4.2.2). This is natural because it allows us to isolate those regions that are densely populated with data points. The reason that we increase the number of $N$ points of a node (line 2) if it has more inherited $Y$ points than $N$ points is to avoid the situation where there may be too few $N$ points left after some cuts or splits. If we fix the number of $N$ points in the entire space to be the number of $Y$ points in the original data, the number of $N$ points at a later node can easily drop to a very small number for a high dimensional space. If there are too few $N$ points, further splits become difficult, but such splits may still be necessary. Figure 5 gives an example.

In Figure 5, the original space contains 32 data $(Y)$ points. According to the above rule, it also has $32 \mathrm{~N}$ points. After two cuts, we are left with a smaller region (region 1). All the $Y$ points are in this region. If we do not increase the number of $N$ points for the region, we are left with only $32 / 2^{2}=8 N$ points in region 1 . This is not so bad because the space has only two dimensions. If we have a very high dimensional space, the number of $N$ points will drop drastically (close to 0 ) after some splits (as the number of $N$ points drops exponentially).

The number of $N$ points is not reduced if the current node is an $N$ node (an $N$ node has more $N$ points than $Y$ points) (line 3 ). A reduction may cause outlier $Y$ points to form $Y$ nodes or regions ( a $Y$ node has an equal number of $Y$ points as $N$ points or more). Then cluster regions and non-cluster regions may not be separated. 


\subsection{Building cluster trees: Two modifications to the decision tree algorithm}

Since the $N$ points are not physically added to the data, we need to make two modifications to the decision tree algorithm in [26] in order to build cluster trees:

1. Compute the number of $N$ points on the fly: From the formulas in Section 2.1 , we see that the gain evaluation needs the frequency or the number of points of each class on each side of a possible cut (or split). Since we do not have the $N$ points in the data, we need to compute them. This is simple because we assume that the $N$ points are uniformly distributed in the space. Figure 6 shows an example. The space has 25 data $(Y)$ points and $25 \mathrm{~N}$ points. Assume the system is evaluating a possible cut $P$. The number of $N$ points on the left-hand-side of $P$ is $25 * 4 / 10=10$. The number of $Y$ points is 3. Likewise, the number of $N$ points on the righthand-side of $P$ is $15(25-10)$, and the number of $Y$ points is 22 . With these numbers, the information gain at $P$ can be computed. Note that by computing the number of $N$ points, we essentially guarantee their uniform distribution.
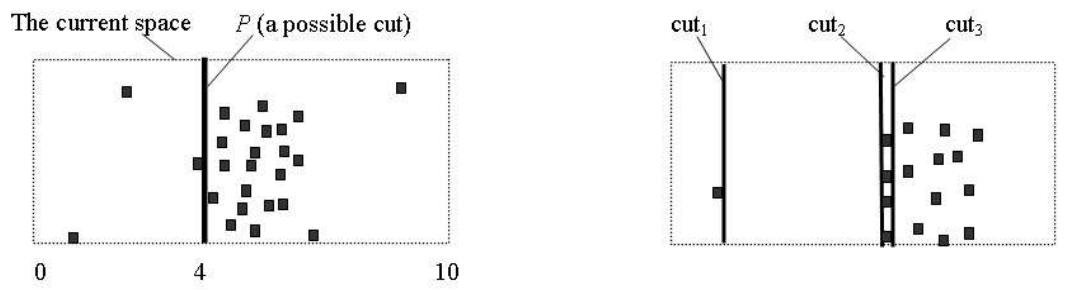

Fig. 6. Computing the number of $N$ Fig. 7. Cutting on either side of data points points

2. Evaluate on both sides of data points: In the standard decision tree building, cuts only occur on one side of data points [26]. However, for our purpose, this is not adequate as the example in Figure 7 shows. Figure 7 gives 3 possible cuts. cut $_{1}$ and cut $_{3}$ are on the right-hand-side of some data points, while cut $_{2}$ is on the left-hand-side. If we only allow cuts on the right-hand-side of data points, we will not be able to obtain a good cluster description. If we use $c_{1} t_{1}$, our cluster will contain a large empty region. If we use $c u t_{3}$, we lose many data points. In this case, $c u t_{2}$ is the best. It cuts on the left-hand-side of the data points. 


\subsection{Building cluster trees: The new criterion for selecting the best cut}

Decision tree building for classification uses the gain criterion to select the best cut. For clustering, this is insufficient. The cut that gives the best gain may not be the best cut for clustering. There are two main problems with the gain criterion:

1. The cut with the best information gain tends to cut into clusters.

2. The gain criterion does not look ahead in deciding the best cut.

Let us see an example. Figure 8(A) shows a space with two clusters, which illustrates the first problem. Through gain computation, we find the best cuts for dimension 1 ( $\left.d_{1 \_} c u t\right)$, and for dimension $2\left(d_{2} \_c u t\right)$ respectively. Clearly, both cuts are undesirable because they cut into clusters. Assume $d_{1}$ ccut gives a better information gain than $d_{2 \_} c u t$. We will use $d_{1 \_}$cut to partition the space. The cluster points on the right of $d_{1 \_} c u t$ from both clusters are lost.

This problem occurs because at cluster boundaries there is normally a higher proportion of $N$ points than that of cluster centers for clusters whose data points follow a normal-like distribution (cluster centers are much denser than boundaries) as we assume that the $N$ points are uniformly distributed in the entire area. The gain criterion will find a balanced point for partitioning, which tends to be somewhere inside the clusters.

Next, we look at the second problem using Figure 8(B) (same as Figure $8(\mathrm{~A}))$. Ideally, in this situation, we should cut at $d_{2 \_} c u t 2$ or $d_{2 \_} c u t 3$, rather than $d_{1}$ cut (although it gives the highest gain). However, using the gain criterion, we are unable to obtain $d_{2-}$ cut 2 or $d_{2 \_}$cut 3 because the gain criterion does not look ahead to find better cuts. There is also another piece of important information that the gain criterion is unable to capture, the empty region between $d_{2-}$ cut 2 and $d_{2 \_}$cut3. Recognizing the empty region is very important for clustering.

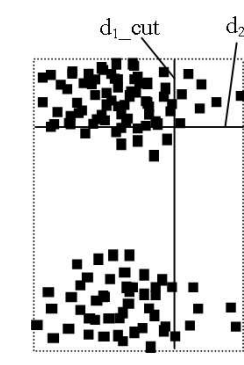

(A)

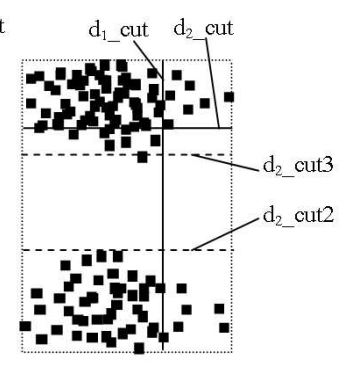

(B)

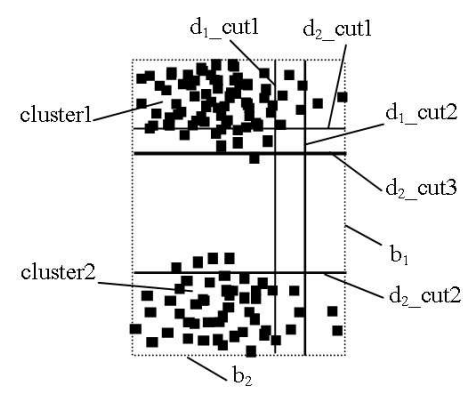

Fig. 8. Problems with the gain criterion

Fig. 9. Determining the best cut

The two problems result in severe fragmentation of clusters (each cluster is cut into many pieces) and loss of data points. To overcome these problems, 
we designed a new criterion, which still uses information gain as the basis, but adds to it the ability to look ahead. We call the new criterion the lookahead gain criterion. For the example in Figure 8(B), we aim to find a cut that is very close to $d_{2}$ cut 2 or $d_{2 \_}$cut 3 .

The basic idea is as follows: For each dimension $i$, based on the first cut found using the gain criterion, we look ahead (at most 2 steps) along each dimension to find a better cut $c_{i}$ that cuts less into cluster regions, and to find an associated region $r_{i}$ that is relatively empty (measured by relative density, see below). $c_{i}$ of the dimension $i$ whose $r_{i}$ has the lowest relative density is selected as the best cut. The intuition behind this modified criterion is clear. It tries to find the emptiest region along each dimension to separate clusters.

Definition (relative density): The relative density of a region $r$ is computed with $r . Y / r . N$, where $r . Y$ and $r . N$ are the number of $Y$ points and the number of $N$ points in $r$ respectively. We use the example in Figure 9 (a reproduction of Figure 8(A)) to introduce the lookahead gain criterion. The algorithm is given in Figure 10. The new criterion consists of 3 steps:

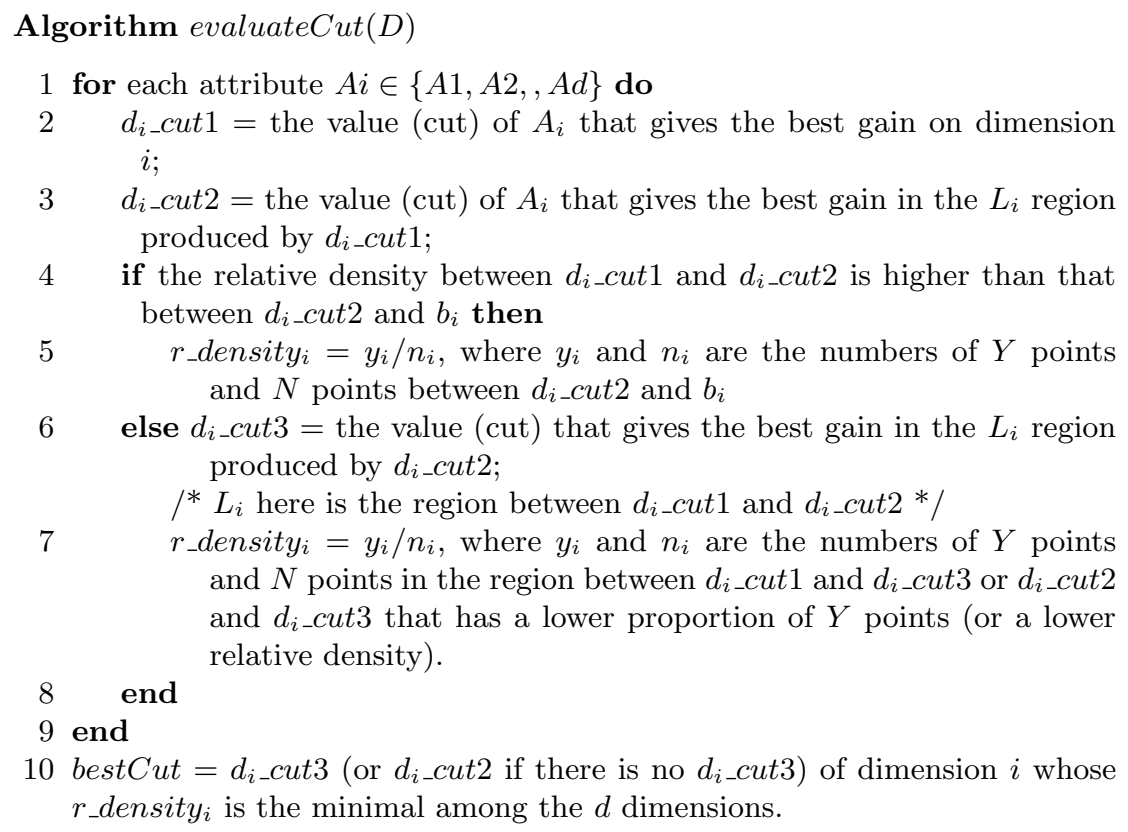

Fig. 10. Determining the best cut in CLTree

1. Find the initial cuts (line 2, Figure 10): For each dimension $i$, we use the gain criterion to find the first best cut point $d_{i_{-}} c u t 1$. For example, in Figure 9 , for dimension 1 and 2, we find $d_{1 \_} c u t 1$, and $d_{2 \_}$cut 1 respectively. 
If we cannot find $d_{i-}$ cut 1 with any gain for a dimension, we ignore this dimension subsequently.

2. Look ahead to find better cuts (lines 3 and 6, Figure 10): Based on the first cut, we find a better cut on each dimension by further gain evaluation. Our objectives are to find:

a) a cut that cuts less into clusters (to reduce the number of lost points), and

b) an associated region with a low relative density (relatively empty).

Let us denote the two regions separated by $d_{i-}$ cut 1 along dimension $i$ as $L_{i}$ and $H_{i}$, where $L_{i}$ has a lower relative density than $H_{i} . d_{i-} c u t 1$ forms one boundary of $L_{i}$ along dimension $i$. We use $b_{i}$ to denote the other boundary of $L_{i}$. To achieve both $2 \mathrm{a}$ and $2 \mathrm{~b}$, we only find more cuts (at most two) in $L_{i}$. We do not go to $H_{i}$ because it is unlikely that we can find better cuts there to achieve our objectives (since $H_{i}$ is denser). This step goes as follows:

Along each dimension, we find another cut $\left(d_{i-}\right.$ cut 2$)$ in $L_{i}$ that gives the best gain. After $d_{i_{-}} c u t 2$ is found, if the relative density of the region between $d_{i_{-}} c u t 1$ and $d_{i_{-}} c u t 2$ is higher than that between $d_{i_{-}} c u t 2$ and $b_{i}$, we stop because both objectives are achieved. If not, we find the third cut $\left(d_{i-c u t} 3\right)$ by applying the same strategy. We seek the additional cut in this case because if the region between $d_{i_{-}} c u t 2$ and $b_{i}$ is denser, it means that there may be clusters in that region. Then, $d_{i-}$ cut 2 is likely to cut into these clusters.

For example, in Figure 9, we first obtain $d_{1 \_} c u t 2$ and $d_{2}$ cut 2 . Since the relative density of the region between $d_{1 \_} c u t 1$ and $d_{1 \_} c u t 2$ is higher than that between $d_{1 \_}$cut 2 and the boundary on the right $\left(b_{1}\right)$, we stop for dimension 1 . We have found a better cut $d_{1 \_}$cut 2 and also a low density region between $d_{1 \_}$cut 2 and the right space boundary $\left(b_{1}\right)$.

However, for dimension 2, we now obtain a situation (in the region between $d_{2-c u t} 1$ and the bottom space boundary, $b_{2}$ ) like that for dimension 1 before $d_{1 \_} c u t 2$ is found. $d_{2 \_} c u t 2$ cuts into another cluster. We then apply the same method to the data points and the region between $d_{2-}$ cut 1 and $d_{2 \_}$cut2 since the relative density is lower between them, another local best cut $d_{2 \_}$cut 3 is found, which is a much better cut, i.e., cutting almost at the cluster boundary. We now have two good cuts $d_{1 \_}$cut2 and $d_{2 \_}$cut3 for dimension 1 and 2 respectively. We also found two low density regions associated with the cuts, i.e., the region between $d_{1 \_}$cut 2 and the right space boundary $\left(b_{1}\right)$ for dimension 1 , and the region between $d_{2 \_} c u t 2$ and $d_{2 \_}$cut 3 for dimension 2 .

3. Select the overall best cut (line 5, 7 and 10): We compare the relative densities $\left(r_{-}\right.$density $\left.{ }_{i}\right)$ of the low density regions identified in step 2 of all dimensions. The best cut in the dimension that gives the lowest $r_{-}$densityi value is chosen as the best cut overall. In our example, the relative density between $d_{2 \_}$cut 2 and $d_{2 \_}$cut 3 is clearly lower than that between $d_{1 \_}$cut 2 and the right space boundary, thus $d_{2 \_}$cut 3 is the overall best cut. 
The reason that we use relative density to select the overall best cut is because it is desirable to split at the cut point that may result in a big empty $(N)$ region (e.g., between $d_{2 \_} c u t 2$ and $d_{2 \_} c u t 3$ ), which is more likely to separate clusters.

This algorithm can also be scaled up using the existing decision tree scaleup techniques in $[18,27]$ since the essential computation here is the same as that in decision tree building, i.e., the gain evaluation. Our new criterion simply performs the gain evaluation more than once.

\section{User-Oriented Pruning of Cluster Trees}

The recursive partitioning method of building cluster trees will divide the data space until each partition contains only points of a single class, or until no test (or cut) offers any improvement ${ }^{5}$. The result is often a very complex tree that partitions the space more than necessary. This problem is the same as that in classification [26]. There are basically two ways to produce simpler trees:

1. Stopping: deciding not to divide a region any further, or

2. Pruning: removing some of the tree parts after the tree has been built.

The first approach has the attraction that time is not wasted in building the complex tree. However, it is known in classification research that stopping is often unreliable because it is very hard to get the stopping criterion right [26]. This has also been our experience. Thus, we adopted the pruning strategy. Pruning is more reliable because after tree building we have seen the complete structure of data. It is much easier to decide which parts are unnecessary.

The pruning method used for classification, however, cannot be applied here because clustering, to certain extent, is a subjective task. Whether a clustering is good or bad depends on the application and the user's subjective judgment of its usefulness $[9,24]$. Thus, we use a subjective measure for pruning. We use the example in Figure 11 to explain.

The original space is partitioned into 14 regions by the cluster tree. By simply looking at Figure 11, it is not clear whether we should report one cluster (the whole space) or two clusters. If we are to report two clusters, should we report the region $\mathrm{C} 1$ and $\mathrm{C} 2$, or S1 and S2? The answers to these questions depend on the specific application.

We propose two interactive approaches to allow the user to explore the cluster tree to find meaningful/useful clusters.

Browsing: The user simply explores the tree him/herself to find meaningful clusters (prune the rest). A user interface has been built to facilitate this exploration. This is not a difficult task because the major clusters are identified

\footnotetext{
${ }^{5}$ We use the same criterion as that in [26] to decide whether any improvement can be made.
} 


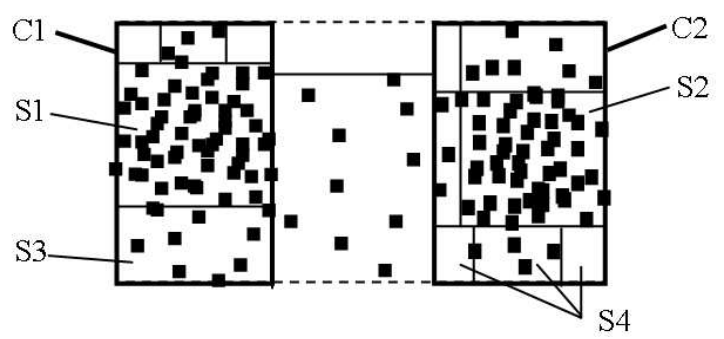

Fig. 11. How many clusters are there, and what are the cluster regions?

at the top levels of the tree.

User-oriented pruning: The tree is pruned using two user-specify parameters (see below). After pruning, we summarize the clusters by extracting only those $Y$ leaves from the tree and express them with hyper-rectangular regions (each $Y$ leaf naturally forms a region or a rule (see Section 2.1)). The user can then view and study them. This is more convenient then viewing a tree, as a tree with many internal nodes can be confusing.

The two parameters used in pruning are as follows:

$\boldsymbol{m i n}_{-} \boldsymbol{y}$ : It specifies the minimal number of $Y$ points that a region must contain (to be considered interesting). min_y is expressed as a percentage of $|D|$. That is, a node with fewer than min_y $*|D|$ number of $Y$ points is not interesting. For example, in Figure 11, if $\min _{-} y *|D|=6$, the number of $Y$ points (which is 4) in S4 (before it is cut into three smaller regions) is too few. Thus, further cuts will not be considered, i.e., the two cuts in S4 are pruned. However, S4 may join S2 (see below) to form a bigger cluster.

$\boldsymbol{m i n} \_\boldsymbol{r d}$ : It specifies whether an $N$ region (node) $E$ should join an adjacent $Y$ region $F$ to form a bigger cluster region. If the relative density of $\mathrm{E}$, E.Y/E.N, is greater than min_rd, where E.Y (or E.N) gives the number of $Y$ (or $N$ ) points contained in $E$, then $E$ and $F$ should be combined to form a bigger cluster. For example, the min_rd value will decide whether S3 should join S1 to form a bigger cluster. If so, more data points are included in the cluster.

The pruning algorithm is given in Figure 12. The basic idea is as follows: It recursively descends down the tree in a depth first manner and then backs up level-by-level to determine whether a cluster should be formed by a $Y$ node alone or by joining it with the neighboring node. If the two subtrees below a node can be pruned, the algorithm assigns TRUE to the Stop field (node.Stop) of the node data structure. Otherwise, it assigns FALSE to the field.

Once the algorithm is completed, we simply descend down the tree again along each branch to find the first $Y$ node whose Stop field is TRUE (not shown in Figure 12). These nodes are the clusters, which are presented to the user as a list of cluster regions. 


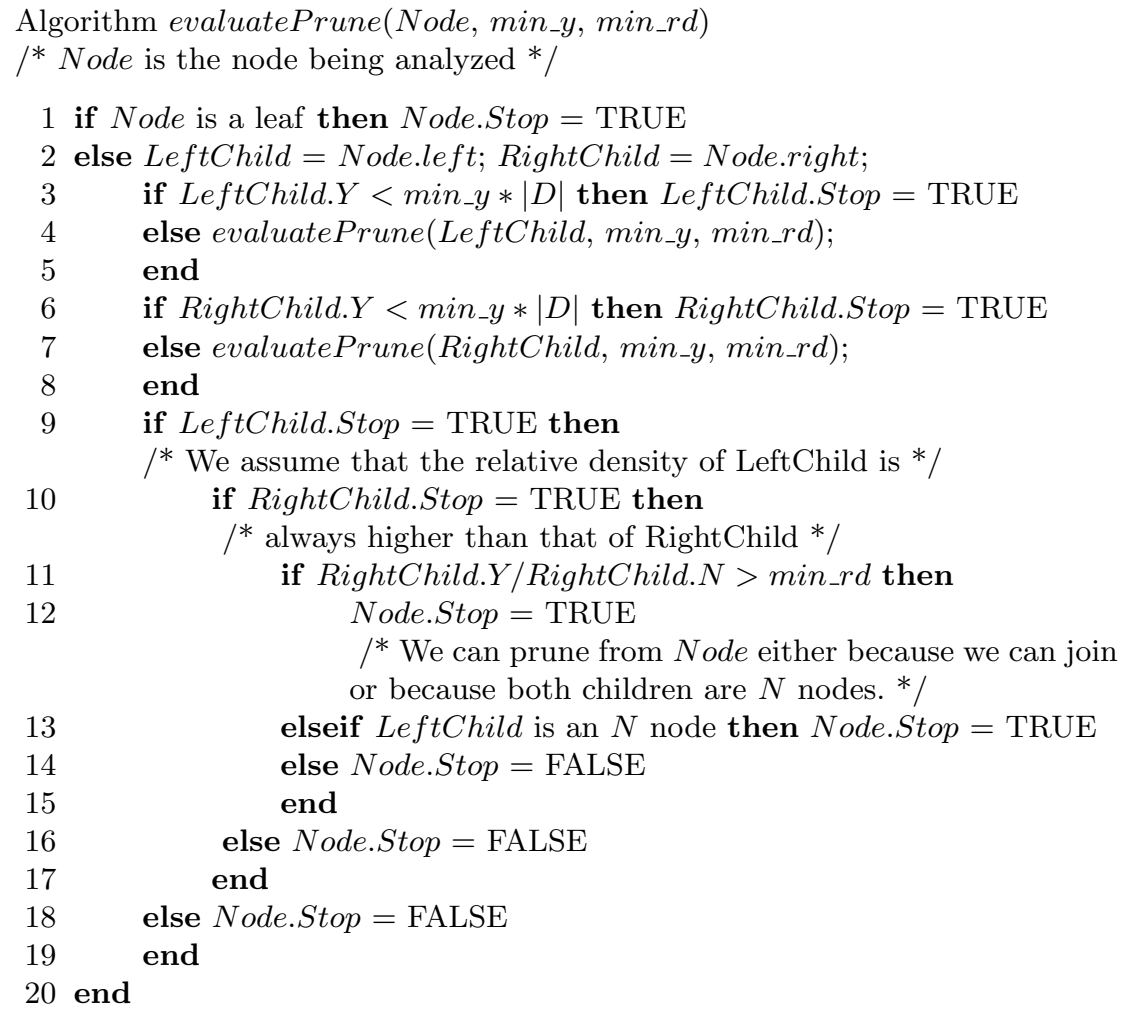

Fig. 12. The cluster tree pruning algorithm

The evaluatePrune algorithm is linear to the number of nodes in the tree as it traverses the tree only once.

\section{Performance Experiments}

In this section, we empirically evaluate CLTree using synthetic as well as real-life datasets. Our experiments aim to establish the following:

- Efficiency: Determine how the execution time scales with, dimensionality of clusters, size of datasets, dimensionality of datasets, and number of clusters in the data space.

- Accuracy: Test if CLTree finds known clusters in subspaces as well as in the full space of a high dimensional space. Since CLTree provides descriptions of clusters, we test how accurate the descriptions are with different pruning parameters. We also test how the ratio of the numbers of $N$ and $Y$ points affect the accuracy. 
All the experiments were run on SUN E450 with one 250MHZ cpu and 512MB memory.

\subsection{Synthetic data generation}

We implemented two data generators for our experiments. One generates datasets following a normal distribution, and the other generates datasets following a uniform distribution. For both generators, all data points have coordinates in the range $[0,100]$ on each dimension. The percentage of noise or outliers (noise level) is a parameter. Outliers are distributed uniformly at random throughout the entire space.

Normal distribution: The first data generator generates data points in each cluster following a normal distribution. Each cluster is described by the subset of dimensions, the number of data points, and the value range along each cluster dimension. The data points for a given cluster are generated as follows: The coordinates of the data points on the non-cluster dimensions are generated uniformly at random. For a cluster dimension, the coordinates of the data points projected onto the dimension follow a normal distribution. The data generator is able to generate clusters of elliptical shape and also has the flexibility to generate clusters of different sizes.

Uniform distribution: The second data generator generates data points in each cluster following a uniform distribution. The clusters are hyperrectangles in a subset (including the full set) of the dimensions. The surfaces of such a cluster are parallel to axes. The data points for a given cluster are generated as follows: The coordinates of the data points on non-cluster dimensions are generated uniformly at random over the entire value ranges of the dimensions. For a cluster dimension in the subspace in which the cluster is embedded, the value is drawn at random from a uniform distribution within the specified value range.

\subsection{Synthetic data results}

\section{Scalability results}

For our experiments reported below, the noise level is set at $10 \%$. The execution times (in sec.) do not include the time for pruning, but only tree building. Pruning is very efficient because we only need to traverse the tree once. The datasets reside in memory.

Dimensionality of hidden clusters: CLTree can be used for finding clusters in the full dimensional space as well as in any subspaces. Figure 13 shows the scalability as the dimensionality of the clusters is increased from 2 to 20 in a 20-dimensional space. In each case, 5 clusters are embedded in different subspaces of the 20-dimensional space. In the last case, the clusters are in the full space. Each dataset has 100,000 records. From the figure, we see that when the clusters are hyper-rectangles (in which the data points 
are uniformly distributed), CLTree takes less time to build the tree. This is because CLTree can naturally find hyper-rectangular clusters, and thus tree building stopped earlier. For both normal and uniform distribution data, we obtain better than linear scale-up.
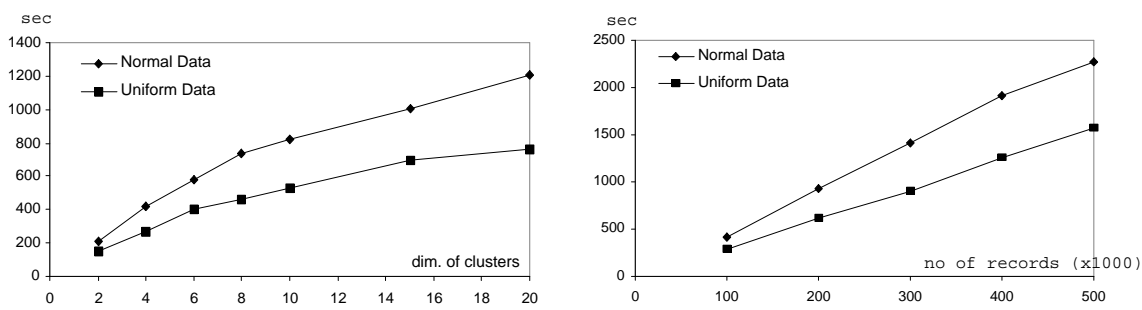

Fig. 13. Scalability with the dimensionality of hidden clusters

Fig. 14. Scalability with the dataset size

Dataset size: Figure 14 shows the scalability as the size of the dataset is increased from 100,000 to 500,000 records. The data space has 20 dimensions, and 5 hidden clusters, each in a different 5 -dimensional subspace. The execution time scales up linearly (although the time complexity of the algorithm is $O(n \log n))$.

Dimensionality of the data space: Figure 15 shows the scalability as the dimensionality of the data space is increased from 10 to 100 . The dataset has 100,000 records and 5 clusters, each in a different 5-dimensional subspace. In both cases (normal distribution data and uniform distribution data), the algorithm scales up linearly.
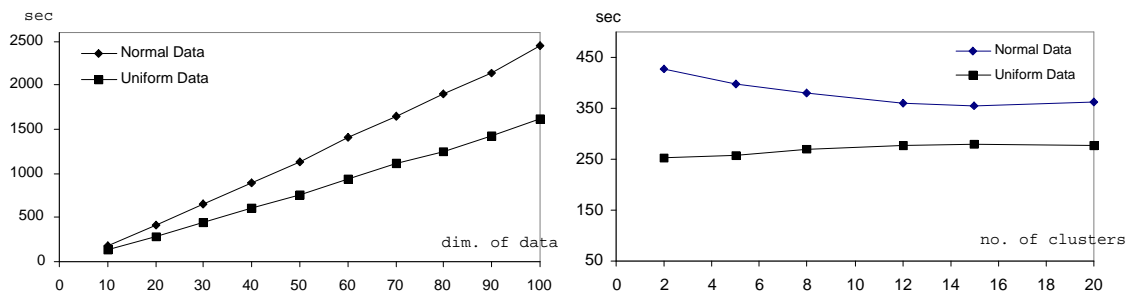

Fig. 15. Scalability with the dimension- Fig. 16. Scalability with the number of ality of the data space clusters

Number of clusters in the space: Figure 16 shows the scalability as the number of clusters in the space is increased from 2 to 20 . The data space has 20 dimensions and 100,000 records. Each cluster is embedded in a different 
5-dimensional subspace. For both uniform and normal datasets, the execution times do not vary a great deal as the number of clusters increases.

\section{Accuracy and sensitivity results}

In all the above experiments, CLTree recovers all the original clusters embedded in the full dimensional space and subspaces. All cluster dimensions and their boundaries are found without including any extra dimension. For pruning, we use CLTree's default settings of $\min \_y=1 \%$ and min_rd $=10 \%$ (see below).

Since CLTree provides precise cluster descriptions, which are represented by hyper-rectangles and the number of data $(Y)$ points contained in each of them, below we show the percentage of data points recovered in the clusters using various min_y and min_rd values, and noise levels. Two sets of experiments are conducted. In both sets, each data space has 20 dimensions, and 100,000 data points. In the first set, the number of clusters is 5, and in the other set, the number of clusters is 10. Each cluster is in a different 5-dimensional subspace. We will also show how the ratio of $N$ and $Y$ points affects the accuracy.

$\boldsymbol{m i n} \_\boldsymbol{y}$ : We vary the value of $\min \_y$ from $0.05 \%$ to $5 \%$, and set min_rd $=10 \%$ and noise level $=10 \%$. Figure 17 gives the results. For uniform distribution, even min_y is very low, all the data points in the clusters are found for both 5 and 10 cluster cases. All the clusters and their dimensions are also recovered. For normal distribution, the percentage of data points found in the clusters is relatively low when min_y is very small $(0.05 \%, 0.1 \%$ or $0.3 \%)$. It increases dramatically and stabilizes after min_y passes $0.5 \%$. From min_y $=$ 0.5 , the percentages of data points (outliers are not counted) recovered are very high, around $95 \%$.
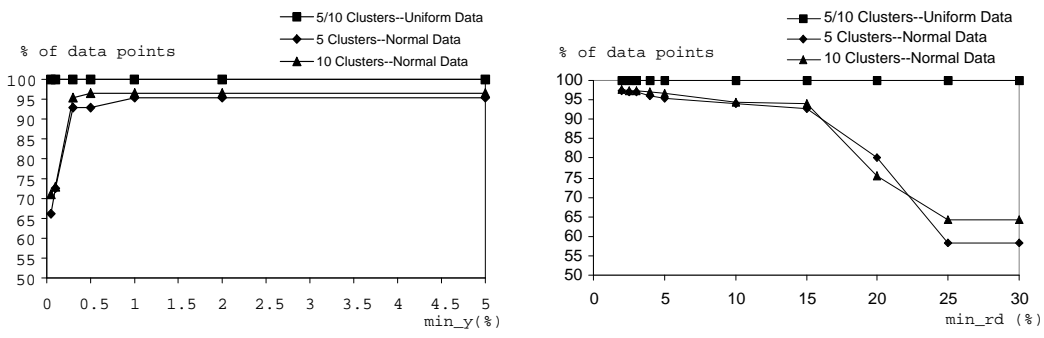

Fig. 17. Percentage of cluster data points Fig. 18. Percentage of cluster data points found with $\min _{-} y$ found with min_rd

min_rd: Figure 18 shows the percentages of data points found in the clusters with different values of min_rd. The noise level is set at $10 \%$ and 
min_y at $1 \%$. The min_rd values in the range of $2-30 \%$ do not affect the number of cluster data points found in the uniform distribution datasets. The reason is that clusters in these datasets do not have the problem of low-density regions around the cluster boundaries as in the case of normal distribution.

For the normal distribution datasets, when min_rd is small, more data points are found, $95 \%$ or more. When min_rd is too small, i.e., below $2 \%$ (not shown in Figure 18), some clusters are merged, which is not desirable. When min_rd is more than $15 \%$, the number of data points recovered in the clusters starts to drop and reaches $58-64 \%$ when min_rd is $25 \%$ and $30 \%$. That is, when the min_rd value is very high, we only find the core regions of the clusters. In all these experiments (except those with min_rd below $2 \%$ ), pruning finds the correct number of clusters, and also the cluster dimensions. Only the number of data points contained in each cluster region changes with different min_rd values. The results shown in Figure 18 can be explained using Figure 11 (Section 3). If min_rd is set low, we will find $\mathrm{C} 1$ and $\mathrm{C} 2$. If min_rd is set too low we will find the whole space as one cluster. If it is set very high, we only find S1 and S2.

Noise level: Figure 19 shows how the noise level affects the percentage of data points recovered in the cluster regions (the noise data or outliers are not included in counting). We use min $\_y=1 \%$ and min $\_r d=10 \%$, which are the default settings for pruning as they perform very well. For the uniform distribution datasets, the noise level in the range of $5-30 \%$ does not affect the result. All data points are recovered. For the normal distribution datasets, when the noise level goes up, the percentages of data points found do not change a great deal for both 5 and 10 clusters. In all cases, the clusters are found.

$\boldsymbol{N}: \boldsymbol{Y}$ ratio: In Section 2.2, we assume an equal number of $N$ points as $Y$ points. In fact, 1:1 ratio is not necessary. We experimented with different $N: Y$ ratios (using default min_y and min_rd values, and 10\% noise). The results are shown in Figure 20. In these experiments, all correct clusters are found. The percentage of cluster data points found hardly change.

$\boldsymbol{m i n} \_\boldsymbol{y}$ and $\boldsymbol{m i n} \_\boldsymbol{r d}$ in applications: From the above experiment results, we see that the clustering results are not very sensitive to the min_y and min_rd values. Although when min_rd is too high, we may lose many data points, we can still find the core regions of the clusters. Thus, in a real-life application, it is rather safe to give both parameters high values, e.g., min_y $=1-5 \%$ and min $r d=10-30 \%$. After we have found the core of each cluster, we can lower down the values to find bigger cluster regions. Alternatively, we can explore the cluster tree ourselves from those nodes representing the cluster cores.

\subsection{Real-life data results}

We applied CLTree to three real life datasets. Due to space limitations, we only describe one here. $\min \_y=1 \%$ and $\min \_r d=10 \%$ are used for pruning. 

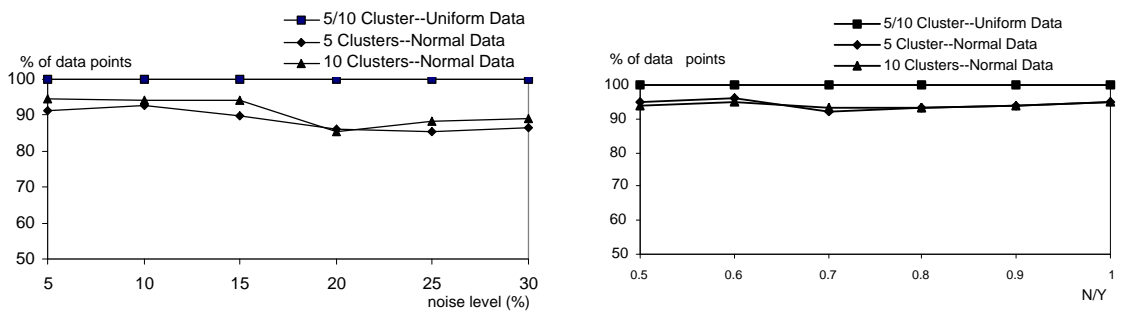

Fig. 19. Percentage of cluster data points Fig. 20. Percentage of cluster data points found with the noise level found with $\mathrm{N}: \mathrm{Y}$ ratio

This dataset is obtained from an educational institution. It stores the examination grades (in A, B, C, D, and F) of their students in 12 courses. CLTree finds 4 main clusters (with some smaller ones). The descriptions of the 4 clusters and the dimensions used are shown in the table below. The last column gives the percentage of students that falls into each cluster. Note that $\mathrm{X}-\mathrm{Y}$ in the table means from grade $\mathrm{X}$ to grade $\mathrm{Y}$.

\begin{tabular}{|c|c|c|c|c|c|c|c|c|c|c|c|c|c|}
\hline Clusters & \multicolumn{10}{|c|}{} & \% of Students \\
\hline & C1 & C2 & C3 & C4 & C5 & C6 & C7 & C8 & C9 & C10 & C11 & C12 & \\
\hline 1 & B-A & C-A & C-A & C-A & C-A & C-A & C-A & C-A & D-A & B-A & C-A & B-A & $14.2 \%$ \\
\hline 2 & & C-A & & & & & & & & F-C & & & $47.7 \%$ \\
\hline 3 & F-C & D-D & F-C & F-D & D-D & F-D & D-D & F-C & & D-D & F-D & F-D & $6.5 \%$ \\
\hline 4 & F-D & F-D & F-D & F-D & F-D & F-C & C-A & & & F-C & & & $8.1 \%$ \\
\hline
\end{tabular}

The first cluster clearly identifies those consistently good students. They perform well in all courses except one, for which they may not do well. It turns out that this course is not a technical course (C9), but a course on writing. There are also two difficult courses (C1 and $\mathrm{C} 10)$ that they do quite well. This cluster is in the full space, involving all 12 dimensions. The value ranges in the 12 dimensions give a precise description of the cluster. Note that although using C10 alone can already separate this cluster from others, we cannot use only B-A for C10 to describe the cluster because it is not precise enough.

The second cluster represents the average student population. Their examination scores can go up and down. This cluster is a subspace cluster, involving only two dimensions. They tend to do reasonably well in $\mathrm{C} 2$ and poorly in C10, which is a difficult course.

Cluster 3 and 4 identify two groups of poor students. Both groups perform badly in courses that require strong logical thinking. However, one group is bad consistently in every course except one, which happens to be the writing course, C9. This cluster is a subspace cluster involving 11 dimensions. The other cluster of students may do reasonably well in those courses that do not 
require strong logical thinking $(\mathrm{C} 8, \mathrm{C} 9, \mathrm{C} 11$, and $\mathrm{C} 12)$. This cluster is also a subspace cluster involving 8 dimensions.

\section{Related Work}

This section consists of two sub-sections. In the first sub-section, we compare CLTree with existing clustering techniques. In the second sub-section, we compare the parameters used in CLTree with those used in existing methods.

\subsection{Clustering techniques}

Traditional clustering techniques can be broadly categorized into partitional clustering and hierarchical clustering [24, 12]. Given n data records, partitional clustering determines a partitioning of the records into $k$ clusters such that the data records in a cluster are nearer to one another than the records in different clusters [24, 12]. The main problem with partitional techniques is that they are often very sensitive to the initial seeds, outliers and the order in which the data points are presented $[24,7,14]$. CLTree does not have these problems.

Hierarchical clustering is a nested sequence of partitions. A clustering can be created by building a tree (also called a dendrogram) either from leaves to the root (agglomerative approach) or from the root down to the leaves (divisive approach) by merging or dividing clusters at each step. Building a tree using either an agglomerative or a divisive approach can be prohibitively expensive for large datasets because the complexity of the algorithms is usually at least $O\left(n^{2}\right)$, where $\mathrm{n}$ is the number of data points or records.

Both partitional and hierarchical methods are based on distance comparison. It is shown in [23] that distance-based approaches are not effective for clustering in a very high dimensional space because it is unlikely that data points are nearer to each other than the average distance between data points $[23,6]$. As a consequence, the difference between the distance to the nearest and the farthest neighbor of a data point goes to zero [6].

CLTree is different from partitional clustering because it does not explicitly group data points using distance comparison. It is different from hierarchical clustering because it does not merge the closest (or split the farthest) groups of records to form clusters. Instead, CLTree performs clustering by classifying data regions ( $Y$ regions) and empty regions ( $N$ regions) in the space using a purity function based on the information theory.

Most traditional clustering methods have very high computational complexities. They are not suitable for clustering of large high dimensional datasets. In the past few years, a number of studies were made to scale up these algorithms by using randomized search (CLARANS [25]), by condensing the data (BIRCH [32] and the system in [7]), by using grids (e.g., DENCLUE 
[22]), and by sampling (CURE [21]). These works are different from ours because our objective is not to scale up an existing algorithm, but to propose a new clustering technique that can overcome many problems with the existing methods.

Recently, some clustering algorithms based on local density comparisons and/or grids were reported, e.g., DBSCAN [10], DBCLASD [31], STING [30], WaveCluster [28] and DENCLUE [22]. Density-based approaches, however, are not effective in a high dimensional space because the space is too sparsely filled (see [23] for more discussions). It is noted in [3] that DBSCAN only runs with datasets having fewer than 10 dimensions. Furthermore, these methods cannot be used to find subspace clusters.

OptiGrid [23] finds clusters in high dimension spaces by projecting the data onto each axis and then partitioning the data using cutting planes at low-density points. The approach will not work effectively in situations where some well-separated clusters in the full space may overlap when they are projected onto each axis. OptiGrid also cannot find subspace clusters.

CLIQUE [3] is a subspace clustering algorithm. It finds dense regions in each subspace of a high dimensional space. The algorithm uses equal-size cells and cell densities to determine clustering. The user needs to provide the cell size and the density threshold. This approach does not work effectively for clusters that involve many dimensions. According to the results reported in [3], the highest dimensionality of subspace clusters is only 10. Furthermore, CLIQUE does not produce disjoint clusters as normal clustering algorithms do. Dense regions at different subspaces typically overlap. This is due to the fact that for a given dense region all its projections on lower dimensionality subspaces are also dense, and get reported. [8] presents a system that uses the same approach as CLIQUE, but has a different measurement of good clustering. CLTree is different from this grid and density based approach because its cluster tree building does not depend on any input parameter. It is also able to find disjoint clusters of any dimensionality.

$[1,2]$ studies projected clustering, which is related to subspace clustering in [3], but find disjoint clusters as traditional clustering algorithms do. Unlike traditional algorithms, it is able to find clusters that use only a subset of the dimensions. The algorithm ORCLUS [2] is based on hierarchical merging clustering. In each clustering iteration, it reduces the number of clusters (by merging) and also reduces the number of dimensions. The algorithm assumes that the number of clusters and the number of projected dimensions are given beforehand. CLTree does not need such parameters. CLTree also does not require all projected clusters to have the same number of dimensions, i.e., different clusters may involve different numbers of dimensions (see the clustering results in Section 4.3).

Another body of existing work is on clustering of categorical data [29, 20, 15]. Since this paper focuses on clustering in a numerical space, we will not discuss these works further. 


\subsection{Input parameters}

Most existing cluster methods critically depend on input parameters, e.g., the number of clusters (e.g., [25, 32, 1, 2, 7]), the size and density of grid cells (e.g., [22, 3, 8]), and density thresholds (e.g., [10, 23]). Different parameter settings often result in completely different clustering. CLTree does not need any input parameter in its main clustering process, i.e., cluster tree building. It uses two parameters only in pruning. However, these parameters are quite different in nature from the parameters used in the existing algorithms. They only facilitate the user to explore the space of clusters in the cluster tree to find useful clusters.

In traditional hierarchical clustering, one can also save which clusters are merged and how far apart they are at each clustering step in a tree form. This information can be used by the user in deciding which level of clustering to make use of. However, as we discussed above, distance in a high dimensional space can be misleading. Furthermore, traditional hierarchical clustering methods do not give a precise description of each cluster. It is thus hard for the user to interpret the saved information. CLTree, on the other hand, gives precise cluster regions in terms of hyper-rectangles, which are easy to understand.

\section{Conclusion}

In this paper, we proposed a novel clustering technique, called CLTree, which is based on decision trees in classification research. CLTree performs clustering by partitioning the data space into data and empty regions at various levels of details. To make the decision tree algorithm work for clustering, we proposed a technique to introduce non-existing points to the data space, and also designed a new purity function that looks ahead in determining the best partition. Additionally, we devised a user-oriented pruning method in order to find subjectively interesting/useful clusters. The CLTree technique has many advantages over existing clustering methods. It is suitable for subspace as well as full space clustering. It also provides descriptions of the resulting clusters. Finally, it comes with an important by-product, the empty (sparse) regions, which can be used to find outliers and anomalies. Extensive experiments have been conducted with the proposed technique. The results demonstrated its effectiveness.

\section{References}

1. C. Aggarwal, C. Propiuc, J. L. Wolf, P. S. Yu, and J. S. Park (1999) A framework for finding projected clusters in high dimensional spaces, SIGMOD-99

2. C. Aggarwal, and P. S. Yu (2000) Finding generalized projected clusters in high dimensional spaces, SIGMOD-00 
3. R. Agrawal, J. Gehrke, D. Gunopulos and P. Raghavan (1998) Automatic subspace clustering for high dimensional data for data mining applications, SIGMOD-98

4. R. Agrawal, S. Ghosh, T. Imielinski, B. Lyer, and A. Swami (1992) In interval classifier for database mining applications, VLDB-92.

5. P. Arabie and L. J. Hubert (1996) An overview of combinatorial data analysis, In P. Arabie, L. Hubert, and G.D. Soets, editors, Clustering and Classification, pages 5-63

6. K. Beyer, J. Goldstein, R. Ramakrishnan and U. Shaft (1999) When is nearest neighbor meaningful?" Proc.7th Int. Conf. on Database Theory (ICDT)

7. P. Bradley, U. Fayyad and C. Reina (1998) Scaling clustering algorithms to large databases, KDD-98

8. C. H. Cheng, A. W. Fu and Y Zhang. "Entropy-based subspace clustering for mining numerical data." KDD-99

9. R. Dubes and A. K. Jain (1976) Clustering techniques: the user's dilemma, Pattern Recognition, 8:247-260

10. M. Ester, H.-P. Kriegal, J. Sander and X. Xu (1996) A density-based algorithm for discovering clusters in large spatial databases with noise, KDD-96

11. M. Ester, H.-P. Kriegel and X. Xu (1995). A database interface for clustering in large spatial data bases, KDD-95

12. B. S. Everitt (1974) Cluster analysis, Heinemann, London

13. C. Faloutsos and K. D. Lin (1995) FastMap: A fast algorithm for indexing, datamining and visualisation of traditional and multimedia datasets, SIGMOD-95

14. U. Fayyad, C. Reina, and P. S. Bradley (1998), Initialization of iterative refinement clustering algorithms, KDD-98.

15. D. Fisher (1987) Knowledge acquisition via incremental conceptual clustering, Machine Learning, 2:139-172

16. K. Fukunaga (1990) Introduction to statistical pattern recognition, Academic Press

17. V. Ganti, J. Gehrke, and R. Ramakrishnan (1999) CACTUS-Clustering categorical data using summaries, KDD-99

18. J. Gehrke, R. Ramakrishnan, V. Ganti (1998) RainForest - A framework for fast decision tree construction of large datasets, VLDB-98

19. J. Gehrke, V. Ganti, R. Ramakrishnan and W-Y. Loh (1999) BOAT - Optimistic decision tree construction, SIGMOD-99.

20. S. Guha, R. Rastogi, and K. Shim (1999) ROCK: a robust clustering algorithm for categorical attributes, ICDE-99,

21. S. Guha, R. Rastogi, and K. Shim (1998) CURE: an efficient clustering algorithm for large databases, SIGMOD-98

22. A. Hinneburg and D. A. Keim (1998) An efficient approach to clustering in large multimedia databases with noise, KDD-98

23. A. Hinneburg and D. A. Keim (1999) An optimal grid-clustering: towards breaking the curse of dimensionality in high-dimensional clustering, VLDB-99

24. A. K. Jain and R. C. Dubes (1988) Algorithms for clustering data, Prentice Hall

25. R. Ng and J. Han (1994) Efficient and effective clustering methods for spatial data mining, VLDB-94.

26. J. R. Quinlan (1992) C4.5: program for machine learning, Morgan Kaufmann

27. J.C. Shafer, R. Agrawal and M. Mehta (1996) SPRINT: A scalable parallel classifier for data mining, VLDB-96. 
28. G. Sheikholeslami, S. Chatterjee and A. Zhang (1998) WaveCluster: a multiresolution clustering Approach for Very Large Spatial Databases, VLDB-98

29. K. Wang, C. Xu, and B Liu (1999) Clustering transactions using large items, CIKM-99.

30. W. Wang, J. Yang and R. Muntz (1997) STING: A statistical information grid approach to spatial data mining, VLDB-97

31. X. Xu, M. Ester, H-P. Kriegel and J. Sander (1998) A non-parametric clustering algorithm for knowledge discovery in large spatial databases, ICDE-98

32. T. Zhang, R. Ramakrishnan and M. Linvy (1996) BIRCH: an efficient data clustering method for very large databases, SIGMOD-96, 103-114 